\title{
Des marges d'Hergé aux secrets des Cités obscures
}

Entretien avec Benoît Peeters

Pierre-Marc de Biasi et Luc Vigier

\section{(2) OpenEdition}

Journals

Édition électronique

URL : http://journals.openedition.org/genesis/1705

DOI : $10.4000 /$ genesis. 1705

ISSN : 2268-1590

Éditeur :

Presses universitaires de Paris Sorbonne (PUPS), Société internationale de génétique artistique littéraire et scientifique (SIGALES)

\section{Édition imprimée}

Date de publication : 12 décembre 2016

Pagination : 133-145

ISBN : 9791023105490

ISSN : $1167-5101$

\section{Référence électronique}

Pierre-Marc de Biasi et Luc Vigier, « Des marges d'Hergé aux secrets des Cités obscures », Genesis [En ligne], 43 | 2016, mis en ligne le 28 novembre 2017, consulté le 30 avril 2019. URL : http:// journals.openedition.org/genesis/1705; DOI : 10.4000/genesis.1705 


\title{
Des marges d'Hergé aux secrets des Cités obscures Entretien avec Benoît Peeters
}

\author{
Propos recueillis par Pierre-Marc de Biasi et Luc Vigier
}

\begin{abstract}
Né en 1956, écrivain, essayiste, biographe et scénariste pour le cinéma et la bande dessinée, Benoît Peeters écrit sur le neuvième art et en particulier sur l'univers d'Hergé depuis plus de trente ans. Animé du souci constant de diffuser et d'expliquer les processus complexes à l'auvre dans la construction de l'image, Benoît Peeters a écrit des textes particulièrement éclairants sur les codes de la bande dessinée ${ }^{1}$, sur Hergé, sur Töpffer et bien d'autres maîtres du dessin contemporains. Coauteur avec François Schuiten de la série monumentale des Cités obscures (seize volumes depuis 1983), il est au contact direct de la pratique du dessin, de l'élaboration des planches, des transactions entre les idées, les pré-visions et les réalisations graphiques. C'est cette dimension, qui apparaît aussi dans certains de ses travaux d'exposition, qu'il a accepté d'évoquer avec nous tout au long de cet entretien.
\end{abstract}

Luc Vigier - Vous vous êtes beaucoup intéressé à la génétique de l'œuvre hergéenne, à travers diverses publications et expositions. Que pouvez-vous nous en dire?

Benoît Peeters - Plus je me suis penché sur l'œuvre d'Hergé, plus j'ai été amené à me plonger dans ses soubassements, à commencer par les variations entre les versions successives des albums. C'est une chose qu'Hergé n'avait pas trop mise en valeur : les albums noir et blanc sont restés longtemps inaccessibles. Et lorsqu'il a redessiné L'Île noire, l'ancienne version couleur a immédiatement disparu. Hergé a rêvé d'une œuvre qui serait perpétuellement synchrone, d'albums qui se trouveraient dans une temporalité imaginaire unique, alors que profondément son œuvre est inscrite dans les événements du XXe siècle. On s'en rend encore mieux compte quand on regarde les versions parues dans la presse. Hergé faisait tout son possible pour effacer cette dimension historique. Il y a chez lui un fantasme d'intemporalité (ou si l'on veut d'éternisation) qu'on peut relier au non-vieillissement des personnages. C'est comme s'il n'était pas supportable que ce Tintin qui a toujours 16 ou 17 ans utilise tantôt des voitures des années trente, tantôt des voitures des années soixante, etc. Il y a donc une première archéologie de l'œuvre qui relie deux temporalités : celle des aventures et celle du travail (et du retravail) d'Hergé, seul puis avec l'équipe des Studios.

La dimension génétique, je l'ai approchée à un second niveau en m'intéressant aux histoires inachevées d'Hergé et notamment en travaillant sur le dossier de Tintin et l'AlphArt. À sa mort, Hergé avait laissé un dossier que l'on m'a demandé de transcrire. J'ai reçu le paquet de notes et d'esquisses, et j'ai tenté de mettre en forme un récit à peu près cohérent, lisible par un assez large public. J'aurais souhaité qu'on publie à la fin du volume une série d'éléments qui ne s'intégraient pas au fil conducteur, des options non retenues, des pistes contradictoires, mais au dernier moment l'éditeur a craint que cela ne paraisse trop compliqué et cette partie a été supprimée. Les tintinophiles ont développé pendant les années suivantes toutes sortes de théories et d'hypothèses sur ce récit inachevé, alors que si on avait disposé des pages de notes et d'esquisses, on aurait vu qu'Hergé envisageait des solutions contradictoires. La transcription des quarante-deux premières pages (à partir de plusieurs variantes pour un certain nombre de pages) n'était à mes yeux en aucun cas la vérité de Tintin et l'Alph-Art. C'était une interprétation du dossier, un dossier encore embryonnaire tant Hergé pouvait modifier la conduite du récit et les dialogues au moment où il se mettait vraiment à dessiner. On devrait réaliser un jour une édition scientifique plus complète et plus ouverte, en n'essayant pas de ramener ce paquet de notes à une linéarité narrative.

1. Case, planche, récit. Comment lire une bande dessinée, Paris, Casterman, 1991; réédité en 2003 chez Flammarion sous le titre Lire la bande dessinée. 
Ce qui m'a passionné encore plus, quelques années plus tard, ce sont les carnets de notes tenus par Hergé à la fin des années trente, puis les recherches pour plusieurs scénarios tardifs, de la fin des années cinquante au début des années soixante-dix. On voit comment son imagination a changé de nature. Au moment des premiers carnets, l'imagination d'Hergé travaille par petites cellules purement affirmatives. Les notations sont brèves, nettes; Hergé sent d'emblée ce qui peut lui servir. Je me souviens par exemple de cette formule : «Requin ou autre animal capturé, révèle l'identité d'un criminel». Plus les années passent, plus Hergé va être gagné par le doute. Tandis que la mélancolie s'impose dans sa vie, il se met à travailler de manière de plus en plus arborescente : désormais, les recherches de scénarios prennent toujours la forme du ou bien/ou bien : Haddock part vers l'Amérique du sud et Tintin l'accompagne ou bien Haddock part seul, tandis que Tintin reste à Moulinsart. S'il reste à Moulinsart, il reçoit rapidement des nouvelles de lui, ou bien il n'en reçoit pas, etc., etc. Or, on sait très bien que tout narrateur qui cultive les arborescences - à moins de faire du Raymond Queneau - est pris rapidement dans un vertige. Tellement de possibles s'ouvrent à chaque étape que le narrateur est saisi par le doute. En observant ces documents de travail, on découvre donc un jeune Hergé, qui travaille d'abord pour la presse et dans la hâte, qui est vraiment dans l'affirmation, semblable à Tintin qui s'élance dans l'escalier en enfilant son imperméable, et court vers le port, puis un Hergé saisi par le doute, qui veut composer un récit, et découvre la prolifération des possibilités narratives. Les arborescences vont peu à peu le paralyser, et en lisant les notes pour les dernières aventures, on a parfois le sentiment qu'il n'a pas choisi le meilleur dans ses propres hypothèses.

Pierre-Marc de Biasi - Qu'est-ce qui a motivé ce changement de stratégie, à votre avis?

B. $P$. - Je suis persuadé qu'il n'y a eu aucune stratégie. Juste une forme de maturation. Et sans doute un sentiment de responsabilité lié au succès grandissant de la série. Parti d'un feuilleton à la petite semaine, dans un hebdomadaire sans grande importance, il évolue peu à peu vers la forme du livre. Dans un premier temps, cela le conduit à se concentrer davantage sur ses histoires, à placer la barre plus haut, graphiquement et narrativement (de Tintin au pays des
Soviets au Lotus bleu, à peine six ans plus tard), les progrès sont extraordinaires. Mais peu à peu, on a l'impression que les difficultés personnelles, mais aussi ce succès qui ne cesse de grandir, ces traductions qui se multiplient, lui font perdre une forme d'évidence. En tant qu'itinéraire d'un créateur, ça m'a beaucoup intéressé, beaucoup troublé et par certains côtés, attristé. En observant de près Hergé à l'œuvre, il me semble qu'on voit tout ce qu' on peut gagner et perdre au fil des ans : le doute, qui l'a fait progresser dans un premier temps, devient à la fin un simple générateur d'angoisse.

\section{P.-M. B. - À partir de quel album?}

B. $P$. - À partir de la fin des années cinquante. Hergé luimême vient d'avoir cinquante ans. Le doute lui permet encore de réaliser deux albums admirables, Tintin au Tibet et Les Bijoux de la Castafiore. Puis la machine s'enraye. Le talent est toujours là, mais la grâce a disparu.

\section{V. - Matériellement, comment se présentent ces esquisses et ces scénarios d'Hergé que vous évoquiez?}

B. $P$. - Au début, ce sont de petits cahiers d'écolier. Plus tard, ce sont des feuilles volantes, souvent du papier à lettres à l'enseigne des Studios Hergé... Le premier carnet que j'ai retrouvé date de 1937, il s'appelle «Éléments Tintin». La première notation c'est : «Trouver un point de départ très simple : le fétiche à l'oreille cassée, trop compliqué». L'Oreille cassée est admirable, à mes yeux comme à ceux de la plupart des tintinophiles, mais Hergé s'était un peu emmêlé les pinceaux en improvisant l'intrigue, semaine après semaine, et il avait eu du mal à la boucler. Il veut donc «trouver un point de départ très simple» : c'est l'amorce de L'île noire. Mais il y a tout de même dans ces «Éléments Tintin» l'idée qu'un récit n'avance pas seulement en ligne droite, qu'il doit contenir des petits modules qui peuvent être des gags, des digressions ou des images particulièrement fortes et inattendues. À cet égard, Hergé est très proche d'Hitchcock et du fameux moulin dont les ailes tournent dans un sens inverse à celui du vent. Même si on est dans une sorte de course au trésor, en poursuivant un «Mac Guffin», il faut éviter tout ce qui rendrait le récit mécanique, il faut construire des séquences autour d'un micro-événement qui perturbe la marche trop rapide et trop prévisible du récit. 
La Mort aux trousses est entièrement construit sur ce mode, tout comme Les Aventures de Tintin.

Ce qui est extrêmement frappant dans ces carnets et ces notes d'Hergé, et que l'on doit retrouver chez d'autres auteurs complets (c'est-à-dire à la fois dessinateur et scénariste), c'est un surgissement graphico-narratif qui prend d'emblée une forme assez proche de la bande dessinée. Il y a des phrases ou des bouts de phrases, des croquis sommaires, parfois une case avec du dialogue, parfois une petite séquence de trois ou quatre images. Il ne s'agit nullement d'un scénario qui attend d'être mis en images. Je me souviens d'une page passionnante intitulée «Milou et les os » : glissant de l'écriture au dessin, de l'os littéral aux os les plus métaphoriques, Hergé esquisse plusieurs gags remarquables dont certains ne seront utilisés que des années après. Il part de l'os de diplodocus que Milou découvre au Musée d'histoire naturelle, tombe sur l'idée d'un drapeau de pirate, puis d'une cabine à haute tension, et arrive à celle des rayons X qui sera utilisée beaucoup plus tard dans Objectif Lune, avec les Dupondt. «Les gags naissent des accidents du crayon», disait remarquablement Hergé dans une de ses premières interviews.

En regardant de telles pages, on comprend à quel point Hergé était fait pour la bande dessinée. Ce qui n'est pas le cas de tous les auteurs, loin de là. J'ai eu l'occasion de feuilleter certains carnets de travail d'Edgar Jacobs, contemporain et ami d'Hergé, dont l'imagination fonctionne de manière très différente. Les Aventures de Blake et Mortimer démarraient par une sorte de petit roman sans le moindre dessin. Ce n'est que dans un deuxième temps que Jacobs découpait son histoire, en numérotant les phrases. Ce processus génétique détermine nettement la structure de récits, littéralement régis par la syntaxe, à coup de «mais», de «tandis que» et de «cependant». Si brillant dessinateur que soit Jacobs, ce ne sont pas les images qui conduisent le récit.

L. V. - Visuellement, comment procède Hergé pour mettre en place les séquences de plus en plus crédibles des Aventures de Tintin? Le document photographique joue-t-il un rôle important dans son travail?

$B$. $P$. - Un rôle colossal. Hergé, qui n'avait pas la possibilité de voyager, a rassemblé une documentation très importante dès le début des années trente : des livres, des photos de films, mais surtout des magazines comme Le Crapouillot et le National Geographic. Il faut parfois un œil de lynx pour reconnaître les documents dont il s'est servi. On m'a montré récemment, dans une revue des années trente, un passant dans un marché, et la manière dont Hergé l'a utilisé, à l'arrière-plan d'une case, pour un personnage tout à fait secondaire. Pour ce qui est des engins et des décors, il s'appuie presque systématiquement sur des photographies à partir du Lotus bleu. Et en 1950, quand il commence la réalisation de On a marché sur la Lune, il achète tout ce qu'on peut trouver à l'époque, même des publications très pointues. Mais ça, beaucoup d'autres auteurs le font, c'est assez normal dans un style réaliste comme le sien. Mais cette idée d'aller chercher une silhouette de personnage dans un marché et de la replacer plusieurs années après dans un coin de case, c'est tout à fait étonnant.

C'est non seulement le signe d'une très grande mémoire visuelle et d'un souci du détail, mais surtout une volonté de constante crédibilité, le désir de mettre en scène un univers plausible, habitable. Je me souviens d'une anecdote d'allure un peu dérisoire qu'Hergé m'avait racontée lors de notre première rencontre : quand il était enfant, il jouait beaucoup avec de petits soldats de plomb assez abîmés; un jour, son oncle lui a offert une boîte contenant d'autres soldats de plomb, tout neufs et plus grands ; Hergé se souvenait n'avoir jamais pu jouer avec eux parce qu'ils n'appartenaient pas au même monde... «On ne pouvait pas les mettre ensemble, m'a-t-il raconté, parce que je n'aurais pas pu y croire.» Si cette anecdote me paraît très révélatrice, c'est parce que ces soldats de plomb ont à peu près l'échelle des personnages dans une case de bande dessinée. L'important pour le lecteur, c'est d'oublier tout de suite que ce sont des soldats de plomb; ils doivent apparaître d'emblée comme une vraie armée, il faut donc que le décor soit adéquat, ni trop défini ni trop flou, qu'il ait le bon degré de réalité. Cette cohérence stylistique, cette unité assurée par la fameuse «ligne claire», c'est quelque chose qu' on ne retrouve jamais dans les films adaptés de Tintin, qu'il s'agisse des dessins animés ou des films avec comédiens. Hergé maîtrise admirablement la bande dessinée, plus que beaucoup d'autres auteurs, parce qu'il n'a jamais été nostalgique d'un autre médium. On sait bien que chez Jacobs, par contre, il y a la nostalgie de l'opéra, la nostalgie du roman populaire, la nostalgie de l'illustration et qu'avec ces trois choses il essaie de bâtir 
un univers. Même s'il le bâtit remarquablement, cela reste un univers composite, fait de beaucoup d'éléments importés. Rien de tel chez Hergé : il était réellement la bande dessinée faite homme.

L. V. - Pouvez-vous évoquer plus précisément le processus de travail d'Hergé, tel que les documents conservés le révèlent?

$B . P$. - Pendant près de vingt-cinq ans, l'essentiel du travail se faisait sur le même support : après une esquisse sommaire, Hergé se mettait à dessiner sur la page définitive, une feuille de papier à dessin d'un format beaucoup plus grand que celui de la publication; il travaillait au crayon, puis à l'encre, en effaçant les traces du crayonné lors de la photogravure. On devine les couches antérieures sur les planches originales, mais seulement à l'état de traces. À partir du moment où Hergé réunit autour de lui une large équipe, c'est-à-dire avec L'Affaire Tournesol en 1954, il met au point une autre méthode, dont il donnera une description précise dans Tintin et moi et de nombreux autres entretiens. Il réalise le crayonné sur une page différente de l'encrage, mais de même format. Le crayonné est désormais très soigné, très poussé, puis Hergé prend au calque les traits qu'il considère comme les meilleurs et les reporte sur la page définitive pour la mise à l'encre. Ce système permet notamment de faire circuler les pages d'un bureau à l'autre : Hergé esquisse la case et détaille les attitudes des personnages, puis ses collaborateurs précisent les décors et les costumes, tracent les phylactères, etc. Le même mécanisme se reproduit au moment de l'encrage : Hergé encre les personnages, mais laisse ses collaborateurs compléter les avions, les décors etc. Vient ensuite la mise en couleurs, sur un nouveau support, au format de publication : Hergé se contente à cette étape de donner quelques indications.

Le seul tort d'Hergé, lorsqu'il a exposé cette méthode, c'est de lui avoir ôté son historicité : il a fait comme s'il avait toujours travaillé de cette manière, alors qu'il avait longtemps presque tout dessiné lui-même, y compris les textes. Certains dessinateurs qui ont voulu par la suite prolonger le style d'Hergé ont imité un processus très tardif, qui a quelque peu rigidifié le style «ligne claire». Au départ, Hergé travaillait de manière beaucoup plus libre. Son dessin se voulait moins réaliste, les noirs y jouaient un grand rôle, et l'épaisseur du trait variait de manière importante. Hergé disait d'ailleurs que lorsqu' on encre un dessin, il faut un trait d'allure égale. Non pas égal, mais «d'allure égale». Il reste toutes sortes de modulations, quasi invisibles mais qui donnent à son dessin une vibration particulière... Il lui arrivait aussi de dire : «il faut encrer en tremblant légèrement». C'est magnifique. Ceux qui ont voulu imiter Hergé ont souvent perdu ce côté légèrement tremblé, cette vitalité spécifique du trait qui conserve quelque chose des hésitations du crayon. Quand Hergé faisait un cadeau à des visiteurs, il donnait les crayonnés, non les mises à l'encre. C'est une époque où il n'y avait pas d'approche génétique de la bande dessinée, peu d'expositions, et pas du tout de marché de la planche originale. Mais il est intéressant de noter que le souvenir qu'il voulait laisser à ceux qui appréciaient son travail, c'était un dessin beaucoup plus bouillonnant et échevelé que les planches définitives. Aujourd'hui, la cote de ces crayonnés est devenue considérable. Heureusement, la plupart ont été conservés, et ils sont exposés par roulement au musée Hergé de Louvain-la-Neuve.

L. V. - Aujourd'hui, les originaux de bande dessinée ont pris une importance considérable. Ils sont exposés régulièrement, atteignent parfois des prix très élevés dans les ventes publiques, etc.

B. $P$. - Oui, il y a désormais une véritable revendication du geste graphique, alors que pendant longtemps on a vu la bande dessinée avant tout comme un récit et les auteurs comme des narrateurs et des humoristes. Le plaisir que procuraient ces histoires empêchait la plupart des lecteurs de les considérer comme des dessins à part entière. Quand je me suis occupé en 1988 avec Pierre Sterckx de la première exposition de ses originaux, «Hergé dessinateur», nombreux sont ceux qui nous ont dit, y compris parmi les passionnés : «je n'imaginais pas que c'était un aussi bon dessinateur». Ils avaient besoin de voir les planches sur le mur, encadrées, isolées, sorties du récit, pour prendre conscience de leur beauté. Moi-même en choisissant les planches avec Pierre, j'ai été émerveillé et j'ai découvert une nouvelle dimension de l'œuvre. Il faut dire aussi que l'original en bande dessinée est généralement beaucoup plus grand que la page publiée, et qu'il révèle de nombreux détails qui ne passaient pas à l'impression. Aujourd'hui, les techniques de reproduction ont beaucoup progressé, ce qui 
a notamment permis le développement de la couleur directe et l'utilisation de nouveaux outils.

\section{P.-M. B. - Beaucoup de ces planches originales gardent aussi des traces du travail préparatoire...}

B. $P$. - Oui, tout à fait, beaucoup d'originaux sont porteurs d'une véritable mémoire de l'élaboration, presque comme des palimpsestes. Même si le crayonné a été en grande partie effacé, on le devine, tout comme les retouches (grattages, collages, retouches à la gouache blanche). Il arrive que cela aille plus loin. Je possède une très belle planche de Blankets, de l'auteur américain Craig Thompson. Pendant la réalisation de ce roman graphique, qui lui a pris cinq ans, il a tenu son journal, au verso de chacune des planches : c'est littéralement l'envers de l'œuvre, une source potentiellement passionnante sur sa genèse. À un degré moindre, les marges des crayonnés d'Hergé sont envahies de petits croquis automatiques, sans rapport avec le contenu de la page; on y trouve aussi des numéros de téléphone, des notes personnelles. Je me souviens d'une planche sur laquelle il avait écrit : «Réserver hôtel Paris. Sous le nom Remi». Pour les spécialistes, ces petites choses sont extrêmement précieuses : elles peuvent aider à dater la page, elles peuvent aussi révéler des sources.

P.-M. B. - Avec François Schuiten, quelle est votre manière de travailler pour les albums des Cités obscures? Cela doit être très différent quand il y a un scénariste et un dessinateur...

B. P. - Oui, mais dans notre cas, les choses sont plus compliquées que ce que peuvent laisser supposer ces deux mots. Nous avons toujours essayé de nous rapprocher de l'heureux état d'auteur complet, qui reste à nos yeux l'idéal en bande dessinée. Nous multiplions donc les discussions avant que je n'écrive un premier synopsis : il faut d'abord que le germe, le concept initial, nous séduise profondément l'un et l'autre, il faut surtout qu'il nous paraisse aussi riche de potentiel graphique que de possibilités narratives. On s'en rend compte très vite : la bonne idée, c'est celle qui ne cesse de rebondir entre nous. Elle peut s'énoncer de manière très simple, comme le trompe-l'œil des Murailles de Samaris, la croissance du réseau de La Fièvre d'Urbicande ou l'inclinaison de Mary dans L'Enfant penchée. Elle peut être plus diffuse, comme dans Brüsel ou La Théorie du grain de sable. Mais il faut vraiment qu'elle s'impose à François comme à moi. J'écris alors un synopsis assez succinct.

Lorsque nous nous lançons dans la réalisation, nous discutons à la table à dessin de la forme que vont prendre les séquences, concevant ensemble la mise en scène et la mise en page. François esquisse rapidement les pages tandis que nous discutons. Et c'est généralement après cette esquisse graphique que j'écris les dialogues. Nous travaillons par blocs de quatre à six pages. Une ou deux semaines plus tard, je viens voir les crayonnés, et nous faisons quelques retouches, dans les textes comme dans les dessins. François n'aurait pas du tout envie que j'écrive un scénario complet, qu'il s'agirait ensuite de réaliser. L'histoire perdrait pour nous de sa vitalité et peut-être de sa nécessité. Nous élaborons le récit de manière semi-feuilletonesque. C'est comme s'il y avait un suspens pour nous en même temps que pour nos lecteurs. Cette méthode n'est pas sans inconvénient et elle peut conduire à quelques moments d'angoisse. Mais en même temps, si on travaille de cette façon depuis trente-cinq ans, c'est que cette technique doit nous convenir.

Il faut se souvenir que la réalisation d'une bande dessinée représente deux ou trois ans pour un dessinateur comme François : il n'a pas d'assistant et réalise absolument tout lui-même, y compris les couleurs. L'aspect artisanal est très conséquent pour un dessinateur. Il est donc essentiel de conserver l'énergie, page après page, case après case. Il y a le plaisir de se surprendre soi-même, mais plus encore le désir de tirer profit de ce qui surgit sur la page.

Quand par exemple, dans La Tour, nous avons introduit la couleur pour les tableaux que découvre notre héros, nous avons été surpris par l'impact que cette idée prenait en se réalisant. Depuis quarante pages, on se trouvait dans un monde noir et blanc et nous voilà confrontés aux premières représentations de cette tour immense. Nous étions presque aussi éberlués que notre héros. Et tout de suite nous avons senti que cet effet devrait réapparaître à la fin de l'histoire. Il y a deux plans de réalité dans l'histoire, et le personnage va passer de l'un à l'autre. Il va entrer dans un monde coloré auquel rien ne l'avait préparé. Ce choc, nous n'aurions pas pu le préméditer abstraitement. Ce qui apparaissait sur la page devenait comme une évidence. Il fallait en tirer parti jusqu'au bout. 


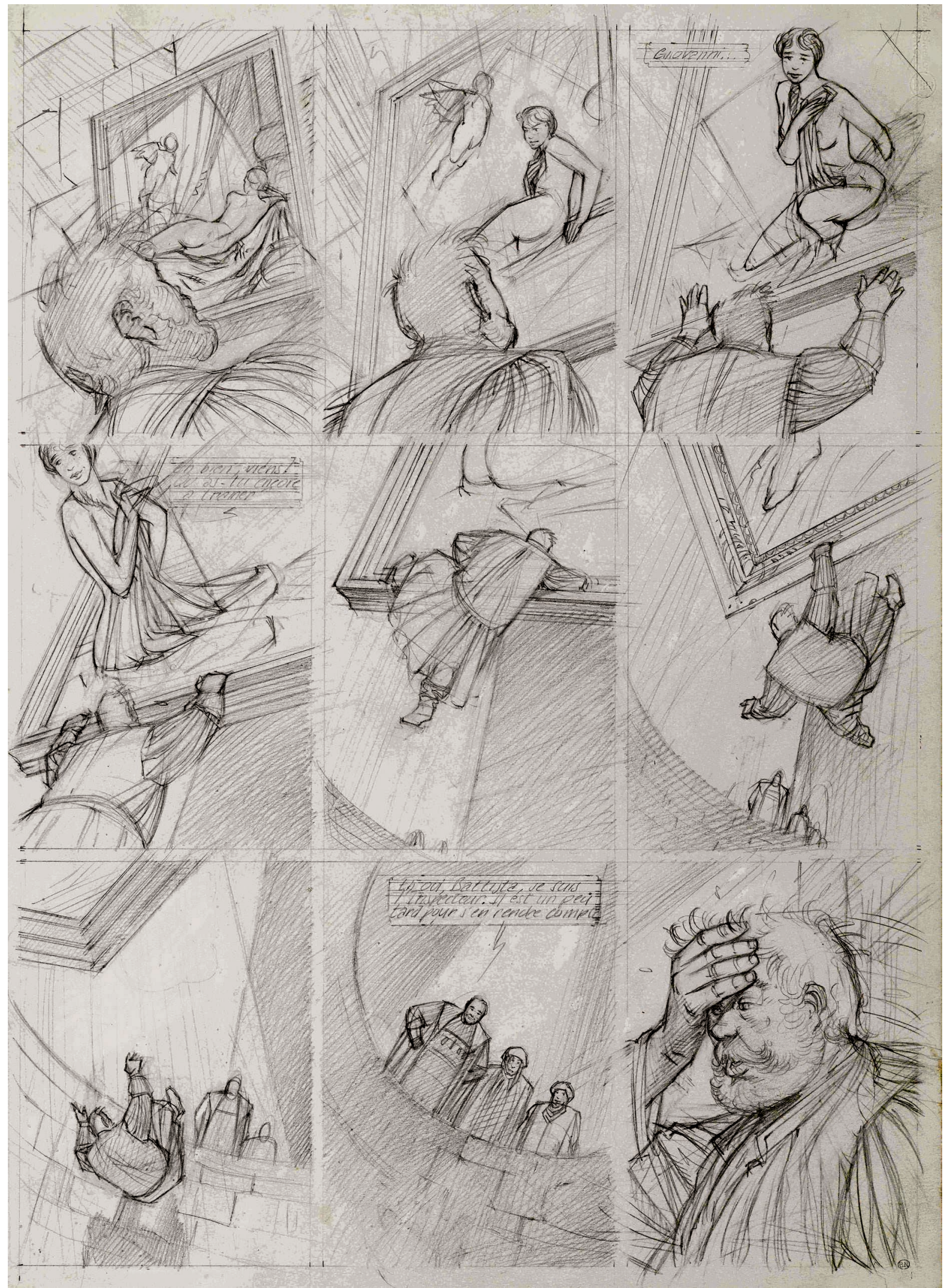



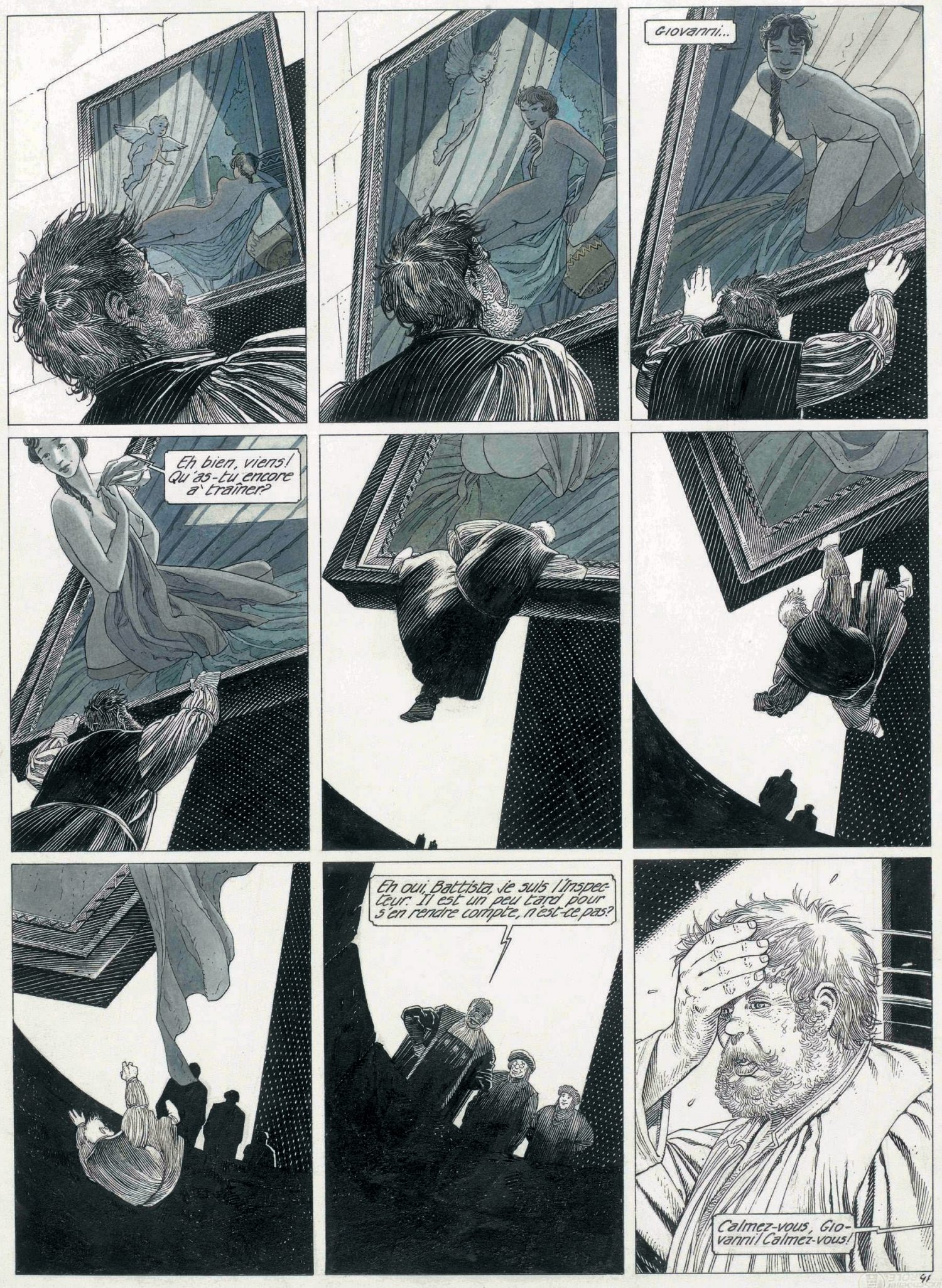

Fig. 2 : La Tour, planche 41 terminée 
$\begin{array}{llllllll}\text { G } & \text { E } & \mathrm{N} & \mathrm{E} & \mathrm{S} & \mathrm{I} & \mathrm{S}\end{array}$

(1) Ar sommet de la Tar, à l'endrat ai la condruchior est abandomere, une bernièe tentatie, taur effilet, minime, continuant de monter dersejeñoñen.

Elle grimpe devant Li, allifre. hei sequie difficilement-Parrener en haut, il comprend la vaniré prefende de la tar, au dele' mino de but ce ofre favait dine Glias C"rsus des sffrts war en arrierà ces quelques pierres, à et esolien inanlant")

Au sommet, contruction al andonnép aprect de chantier - Immenses guas en hais, matériel baraquements.

Is vont $x$ arrin d'ure de ces ques pall descendo is juxpu- an felies suirant.

Ectreme fin = ils émergent par une soute de raterain arse cain de la Tau.

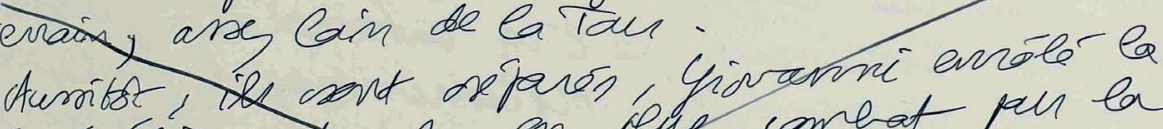
vat os eleimnd de lus on rele, combat pur la rgaindre de e autre

On lien, au dernier imstant, elle retarne dan, $\mathrm{Ca}$ tan fair cherched prelque chose (wo biure al un dyct drelias tas exemple)- Giaranni tuin at la tar or effenteren. Espar fu at puand meime tu etbaffor.

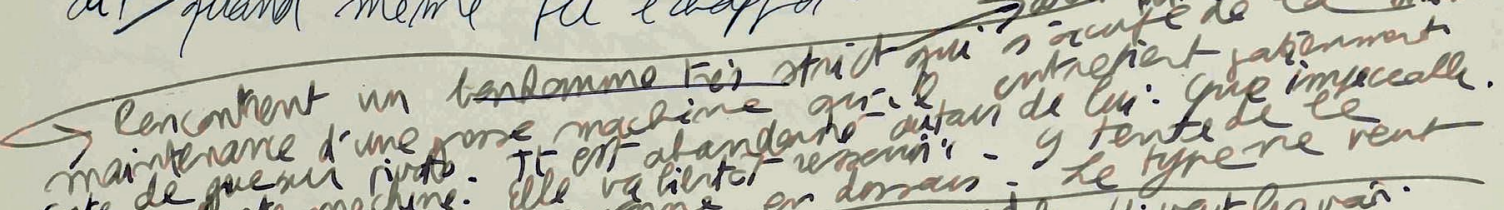

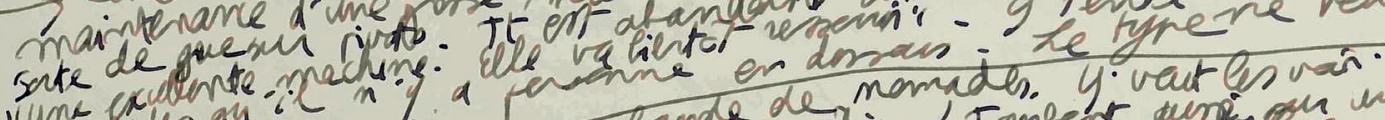

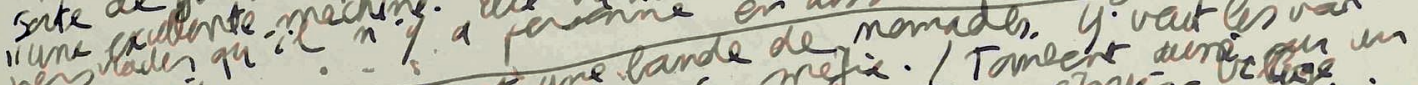
- Viv ovat.

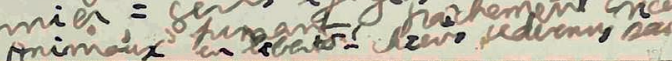

140

Fig. 3 : Benoît Peeters, feuillet du scénario de La Tour

(coll. François Schuiten et Benoît Peeters, Bibliothèque nationale de France, don des auteurs) 
Glle tembrare sardation

vil The vas partin, ntot - ce pas? Jer-en pile, emmene - mai arec tai!

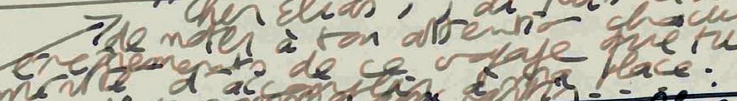
a ac de seicis exhar place.

de ce moment effot de texte ofy-griovanni

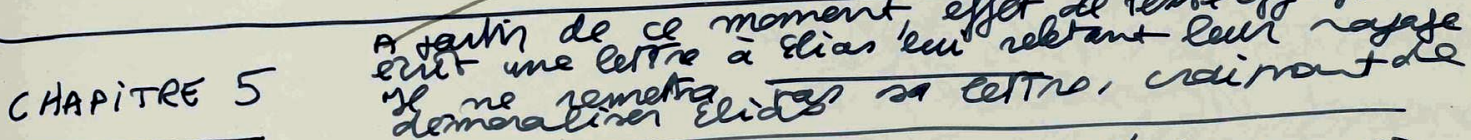

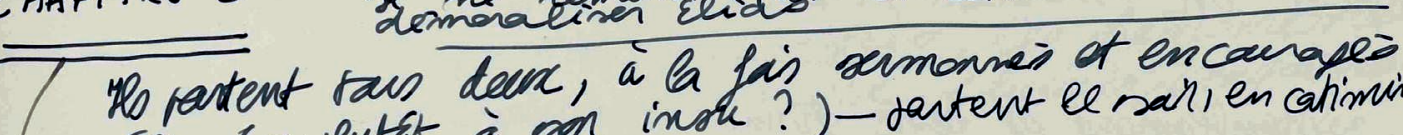

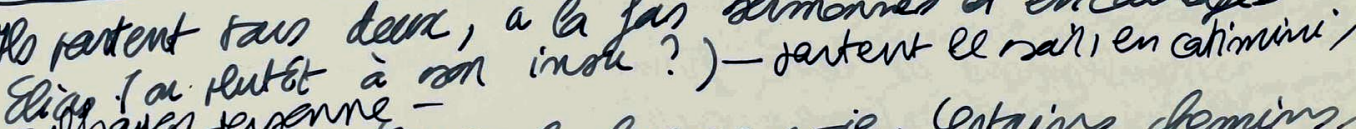
pas cligh rays tersenne-

far nofficule parr traver la Conne wie. Certains chemins.

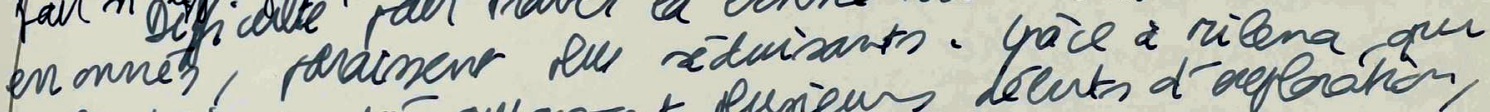

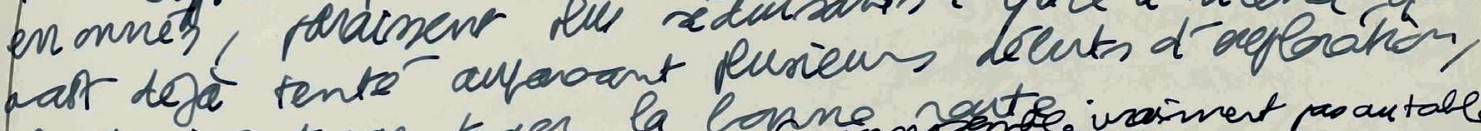

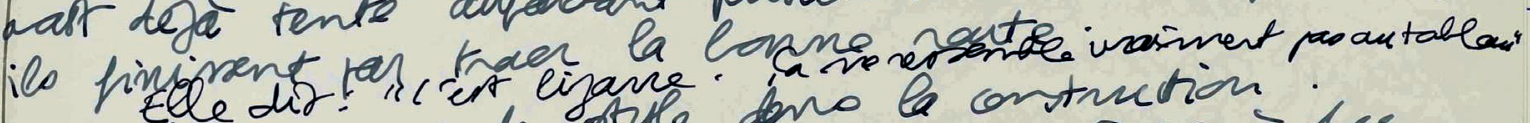
chanjement de style dens la contruction. paraye $a$ un gothigure fambyant, ol merme à lce barapre. Aytartion de techniques

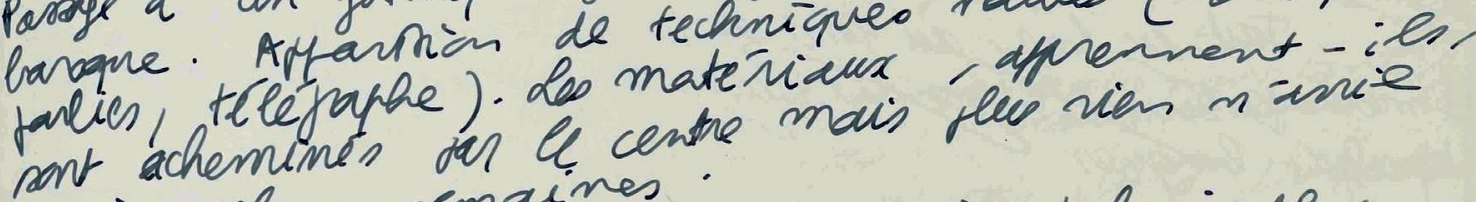
defiin, prelgres remaines

parallitement à cette prgremin techniqué, ondrion plinique - h'idée de la taer er apre l-n

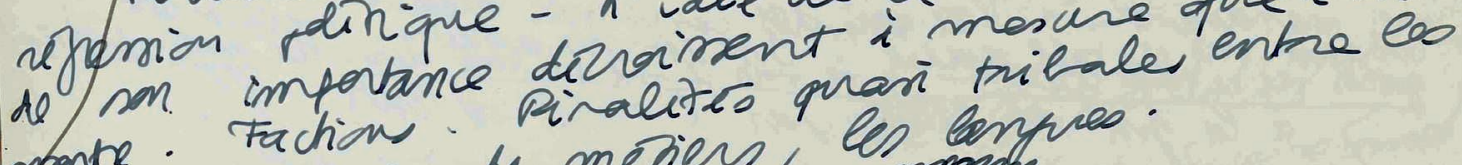

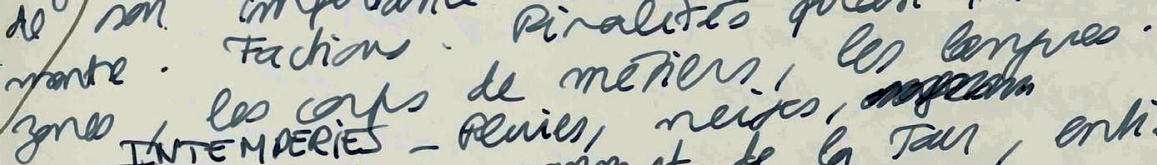
zono IN les corfs - Benies, neiges, sapeesm

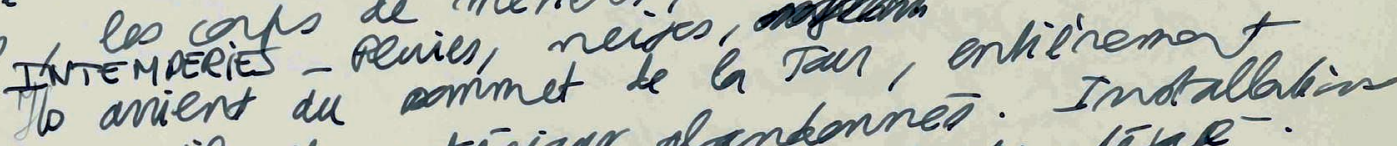
deratt. outils et matériasd alankenneis. Indrallat.

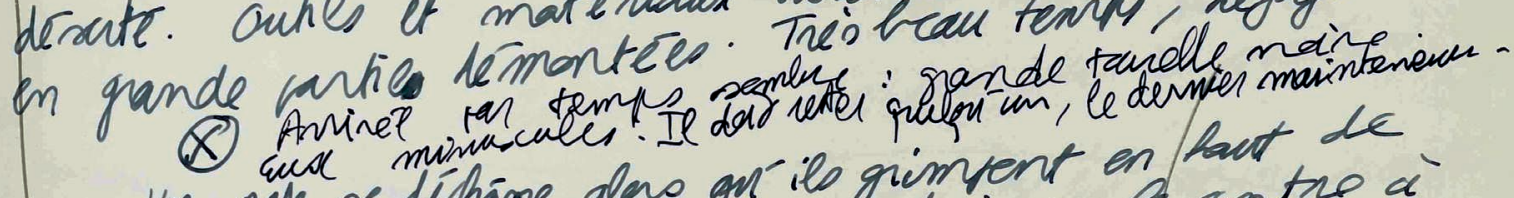

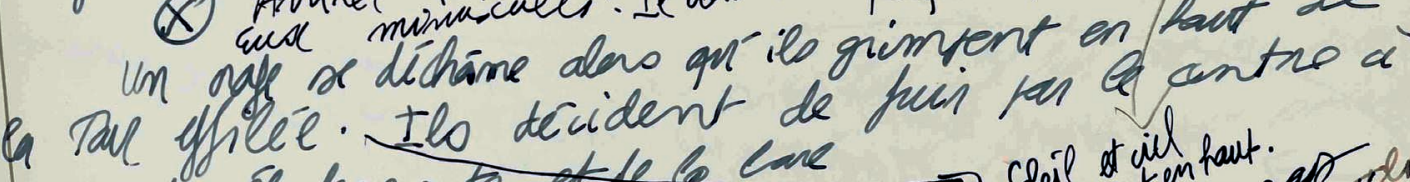

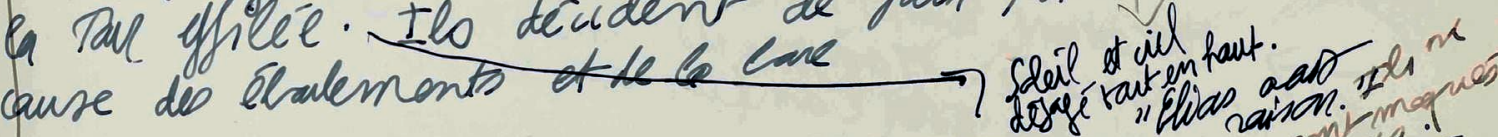

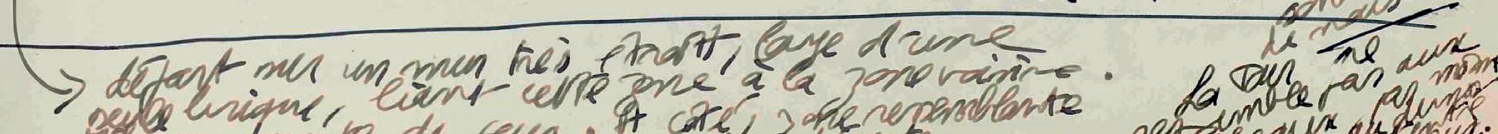

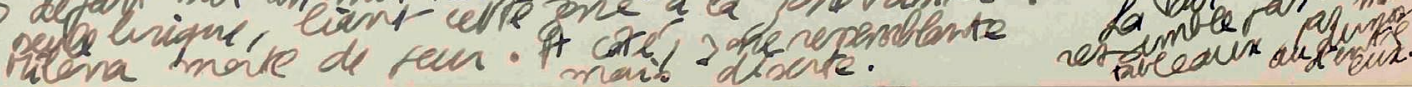


La rencontre du dessin et de la photographie dans l'album L'Enfant penchée obéit à une logique du même ordre : le personnage qui vit dans notre monde, Augustin Desombres, est traité pendant une longue partie de l'histoire sous forme de récit photographique, alors que l'histoire de Mary, la jeune fille inclinée, est traitée comme une bande dessinée classique. Desombres, qui est lui-même peintre, va rejoindre le monde du dessin et y rencontrer la jeune fille. Ils vont vivre une brève histoire d'amour, dans une sorte de no man's land à la croisée des images, avant qu'il n'abandonne Mary. Au dernier instant, elle tente vainement de le retenir en lui attrapant la main. Revenu chez lui et ayant repris un aspect photographique, Augustin Desombres découvre sur sa main des hachures, des stigmates dont il ne pourra jamais se délivrer. Ici non plus, il ne s'agit pas d'une idée littéraire. Bien sûr, tous les éléments étaient en place : la photo, la bande dessinée, la peinture, mais tout d'un coup les choses ont pris, et nous avons senti où l'histoire allait nous conduire. Alors, nous avons allongé cette séquence, ralenti le rythme du découpage, pour faire de ce moment le cœur secret de l'album.

P.-M. B. - Et tout cela suppose un jeu d'allers-retours entre l'écriture et le dessin...

B. P. - Oui, c'est l'une des particularités du travail des Cités obscures, et de notre collaboration. Non seulement, nous sommes des amis d'enfance, mais pendant très longtemps j'ai vécu à Bruxelles, ce qui nous permettait de nous voir très fréquemment. Il y a donc vraiment une création commune. Même si c'est François qui dessine et si c'est moi qui écris les textes, il y a un terrain commun, un entre-deux essentiel qui n'a pas vraiment de nom, mais qui est sans doute la bande dessinée proprement dite.

Bien entendu, dans beaucoup de collaborations scénariste-dessinateur, les choses se passent d'une tout autre manière : Jean-Michel Charlier par exemple travaillait surtout à distance; Jean Van Hamme aussi aujourd'hui, comme beaucoup d'autres. Le scénariste a son terrain de compétence, le dessinateur le sien. Leur travail repose sur la complémentarité des talents, mais aussi sur une forme d'autonomie de chacun. Et cette méthode peut donner d'excellents résultats... Mais avec François, nous avons développé une collaboration amicale, sans chasse gardée, avec de nombreux moments de travail en coprésence. Et notre collaboration s'est rapidement étendue à d'autres terrains que la bande dessinée, où les tâches sont réparties de manière tout à fait différente. Il y a par exemple nos «conférences-fiction», des récits à deux voix où on revisite une histoire en projetant des images : c'est un exercice de semi-improvisation où la parole circule constamment entre nous. Là, nous sommes vraiment dans une collaboration égalitaire et simultanée. Le travail scénographique et les réalisations audiovisuelles ont été aussi des façons d'enrichir la collaboration. Nous avons également beaucoup voyagé ensemble à l'occasion de festivals, d'expositions, de traductions : ce sont des expériences communes, dans un espace-temps assez différent de l'atelier, et ce sont des occasions privilégiées de rêver une future histoire, de manière encore légère...

P.-M. B. - À bien des égards, vous cherchez à fonctionner comme un auteur unique qui s'appellerait "Schuiten Peeters»?

B. P. - Oui, nous avons sans doute une nostalgie de cette confrontation directe avec le médium qui est le propre de l'auteur complet. Il y a - en tout cas, il peut y avoir - dans la bande dessinée quelque chose qui n'est ni le dessin ni le scénario, mais le médium dans toute sa force spécifique, avec les allers-retours constants entre texte et image, narration et figuration, mise en scène et mise en page que cela suppose. Les grands auteurs complets comme Töpffer, McCay, Herriman, Hergé, Fred ou Chris Ware, sont indissociablement scénariste et dessinateur, mais aussi metteur en scène, acteur, décorateur, lettreur, etc. Dans ses premiers écrits sur la bande dessinée, en 1845, Rodolphe Töpffer insiste déjà sur le fait que le «roman en estampes» n'est ni du dessin, ni de l'écriture, ni une simple addition des deux, mais bien une forme neuve, originale, irréductible. Beaucoup d'auteurs complets l'ont senti et ont essayé de le dire. Mais dès qu' on travaille en duo, les gens ont tendance à penser qu'il y a un des auteurs qui a créé l'histoire et l'autre qui l'a mise en images, presque comme s'il en était l'illustrateur. En réalité, dans la collaboration entre deux personnes, il y a beaucoup de figures possibles. Alan Moore par exemple, le scénariste de Watchmen, va extrêmement loin dans la description des images et des pages. On sent qu'il les a imaginées jusque dans leurs plus petits détails, et que, 

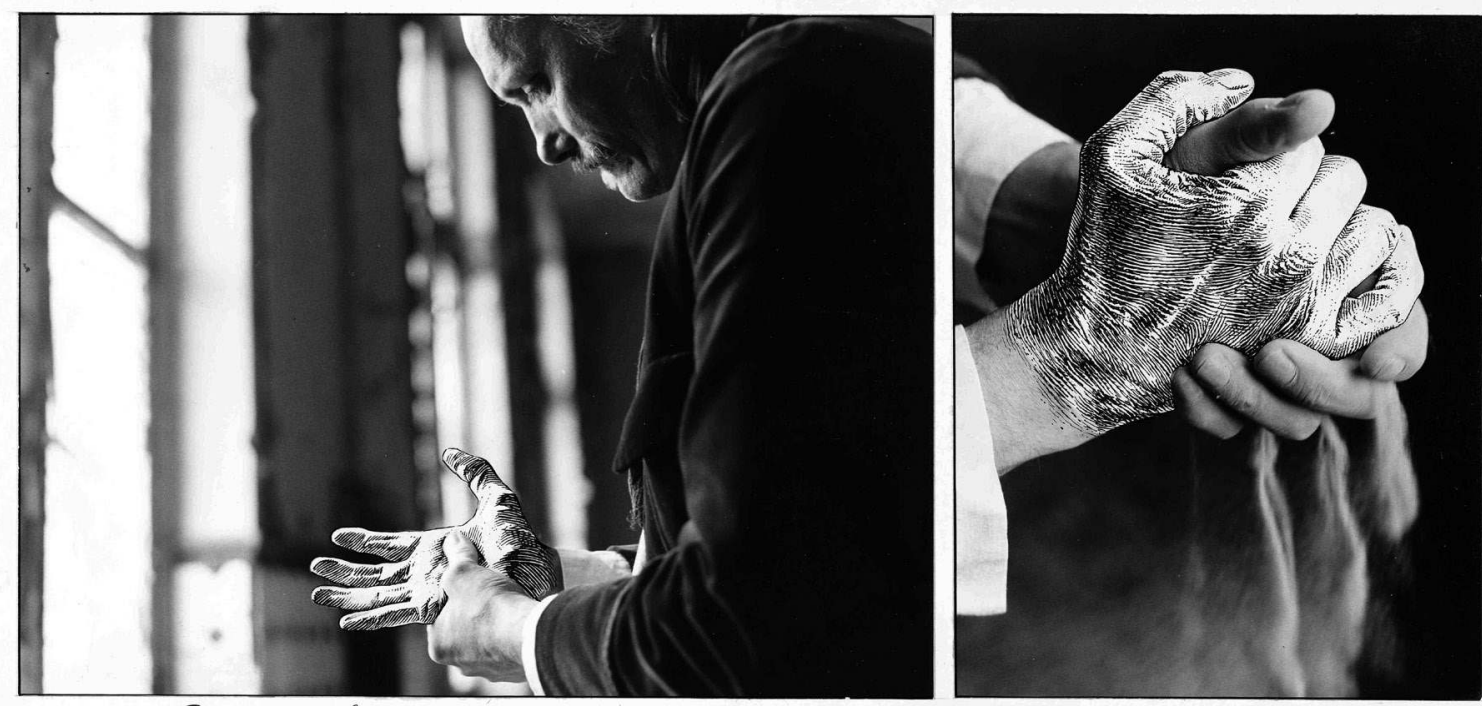

Rien à faire, je poute la trace indelebile de ce moment oxi Mary a pris ma main fwir la retenir dans la sienne. Comme si cette cenconize $m$ 'avait marque an for nouge.

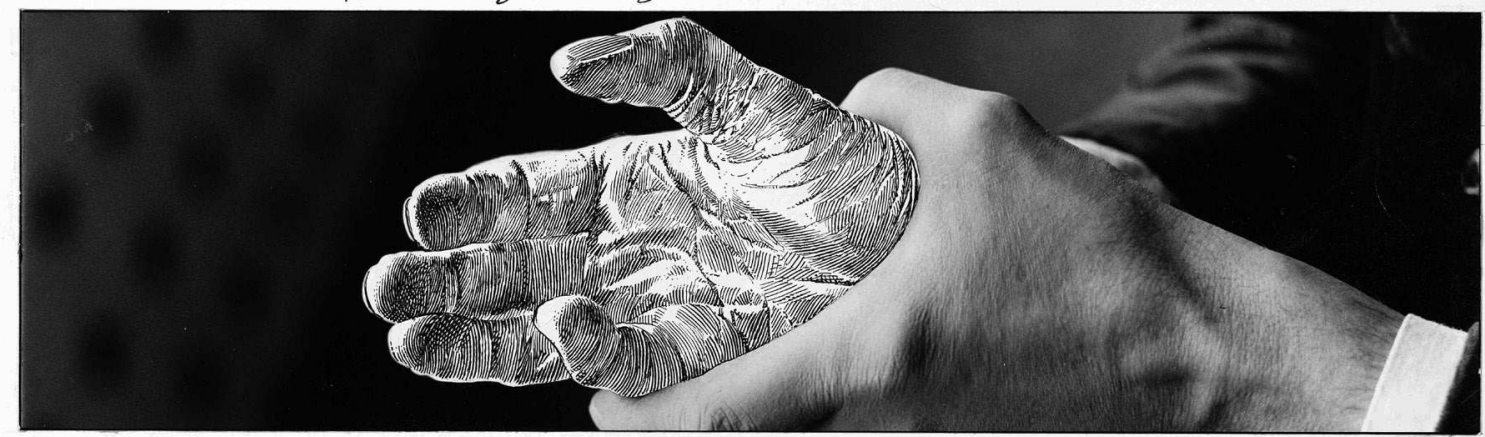

Finalement, Mary, je suis heureux de ne pas être inderno de garder cette trace de tor; ce sowvenir incruste clans ma peau. Ie relalise maintenant combien of ai ete sturide de revenir.

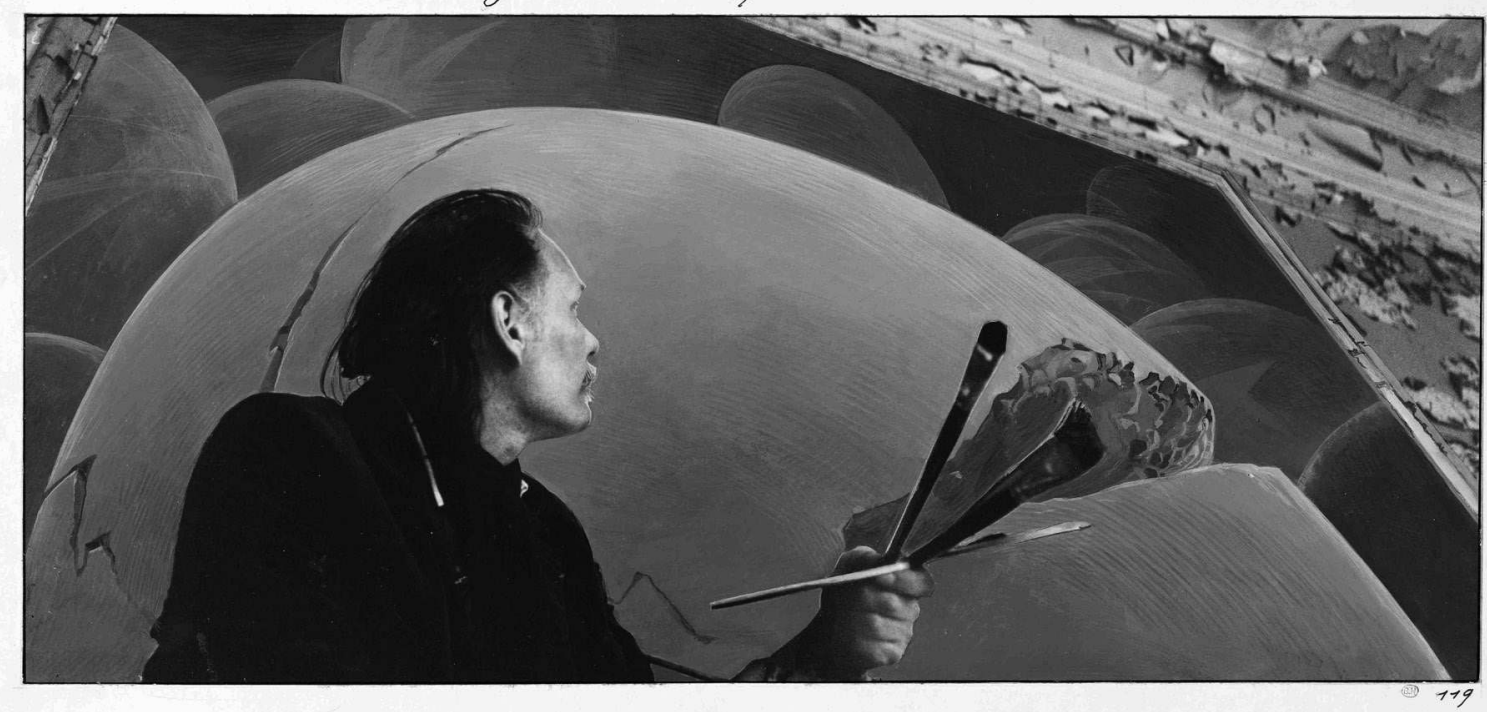

Fig. 4 : L’Enfant penchée, planche 119 
par-delà le récit, il a une conscience incroyable du médium. Mais du coup, je trouve que les dessinateurs avec lesquels il travaille sont un peu contraints d'obéir au scénario. Alors que moi, ce qui me passionne le plus, c'est de donner à celui avec qui je travaille, à chaque moment, le désir de s'investir pleinement dans la page et de la réinventer. Il s'agit de tout autre chose que d'exécuter un programme.

L. V. - Mais dans une collaboration comme la vôtre, est-ce qu'il y a des polarités entre vous deux: des hantises, des obsessions, des figures matricielles?...

B. $P$. - Sûrement, mais nous ne sommes pas les mieux placés pour en parler... Une des questions clés, et une des plus difficiles, c'est sans doute la place de l'inconscient dans les œuvres en collaboration. Quand un écrivain écrit un texte, quand un peintre peint un tableau, il y a, par-delà le programme qu'il s'est donné, un immense travail inconscient qui se fait tout au long de la réalisation : l'œuvre s'invente ou se réinvente entre soi et soi. Dans le cas d'une collaboration, même d'une collaboration très étroite comme celle que j'ai avec François Schuiten, ce processus joue de manière un peu différente. Quelle que soit notre proximité, il y a des éléments que j'ai rêvés et qui se matérialisent chez l'autre sur un autre mode. L'exemple le plus frappant concerne la sexualité et l'érotisme : on peut décrire la beauté d'une femme ou l'intensité d'une scène amoureuse, ce n'est pas pour autant qu'on la voit et qu'on la sent de la même façon que l'autre. Il se passe là quelque chose de tout à fait singulier. Je m'en suis rendu compte dans le travail avec François Schuiten, mais aussi dans les albums que j'ai réalisés avec Frédéric Boilet et avec Marie-Françoise Plissart... Même en dehors d'une composante directement érotique, pour tout ce qui concerne la gestuelle et les mimiques, la relation entre une phrase prononcée et l'expression du visage qui l'accompagne - ce qui au cinéma relèverait du jeu d'acteur -, on est dans quelque chose de très intime. On touche peut-être là au non-dit de la collaboration, ou à son indicible. Quelle que soit la manière dont on décrit une scène ou une attitude, aussi précis que puisse être le scénario, à un moment les choses vont passer par la main et la sensibilité de l'autre. C'est la trace de son corps, de sa relation au monde, de sa relation aux autres. Le sexuel au sens large est vraiment révélateur à cet égard : il est quand même très difficile de croire qu'on va faire partager à l'autre sa vision ou son fantasme. Après avoir beaucoup réfléchi sur la collaboration (j'ai consacré un livre au sujet, Nous est un autre, enquête sur les duos d'écrivains ${ }^{2}$, lui-même écrit en collaboration avec Michel Lafon), c'est une des choses sur lesquelles je continue à m'interroger.

L. V. - Existe-t-il dans votre travail commun des perturbations d'ordre éditorial? Un éditeur qui place des contraintes, des exigences précises en cours de réalisation?

B. $P$. - À cet égard, avec François, nous sommes vraiment des privilégiés. Chez Casterman, dès le début, nous avons fait des choses assez bizarres et on nous a toujours soutenus et laissés libres. Nous avons commencé cette série qui n'en est pas vraiment une par Les Murailles de Samaris, un album d'aspect classique, un quarante-huit pages en couleurs. Mais dès la seconde histoire, La Fièvre d'Urbicande, nous avons fait le choix d'un récit plus long et en noir et blanc, ce qui impliquait à l'époque de publier dans une autre collection, celle des «Romans (à suivre)». Immédiatement après, nous avons fait L'Archiviste, un album de grand format qui n'obéit pas aux codes classiques de la bande dessinée. Puis il y a eu La Tour, publié dans une collection vouée au noir et blanc tout en comportant quelques pages en couleurs. Et ainsi de suite. À chaque fois Casterman nous a suivis et accompagnés. Nous avons parfois dû argumenter, ce qui est normal, mais on ne nous a jamais empêchés de faire ce que nous avions envie de faire. C'est un luxe dont les jeunes auteurs ne bénéficient guère aujourd'hui. Le marché de la bande dessinée est devenu beaucoup plus dur : les revues ont disparu, les albums sont très nombreux et ont bien du mal à se faire une place. Nous-mêmes, il y a certaines expériences que nous n'aurions peut-être plus la possibilité de tenter aujourd'hui.

\section{V. - Les techniques ont beaucoup évolué ces dernières années. Quelle est la place du numérique dans votre travail avec François Schuiten?}

B. $P$. - François ne dessine jamais à la palette graphique. Il a travaillé pour plusieurs projets numériques, mais

2. Paris, Flammarion, 2006 
généralement dans le cadre de films (comme Les Quarxs et $\mathrm{Mr}$ Nobody) ou d'installations (comme Revoir Paris); et même dans ces cas-là, il travaille sur papier. Et pour la bande dessinée, il reste fidèle aux outils qu'il utilise depuis toujours et continue à réaliser les couleurs entièrement à la main. Comme beaucoup de dessinateurs, il est très attaché aux outils et aux supports matériels. François a vu disparaître à regret le papier à dessin qu'il utilisait depuis très longtemps, un papier très épais qui permettait de gratter un élément sans que ça se voie, c'est-à-dire de réencrer de manière absolument invisible. Ces questions très matérielles, les dessinateurs en sont globalement plus conscients que les écrivains... Les dessinateurs adorent parler de leurs outils, de leurs papiers. On peut dire qu'il y a une sorte de primo-matérialisme à cet égard, par opposition au domaine littéraire. Quand deux dessinateurs se rencontrent, ils discutent volontiers de ces aspects artisanaux. Et ils regardent très attentivement les planches originales de leurs collègues.
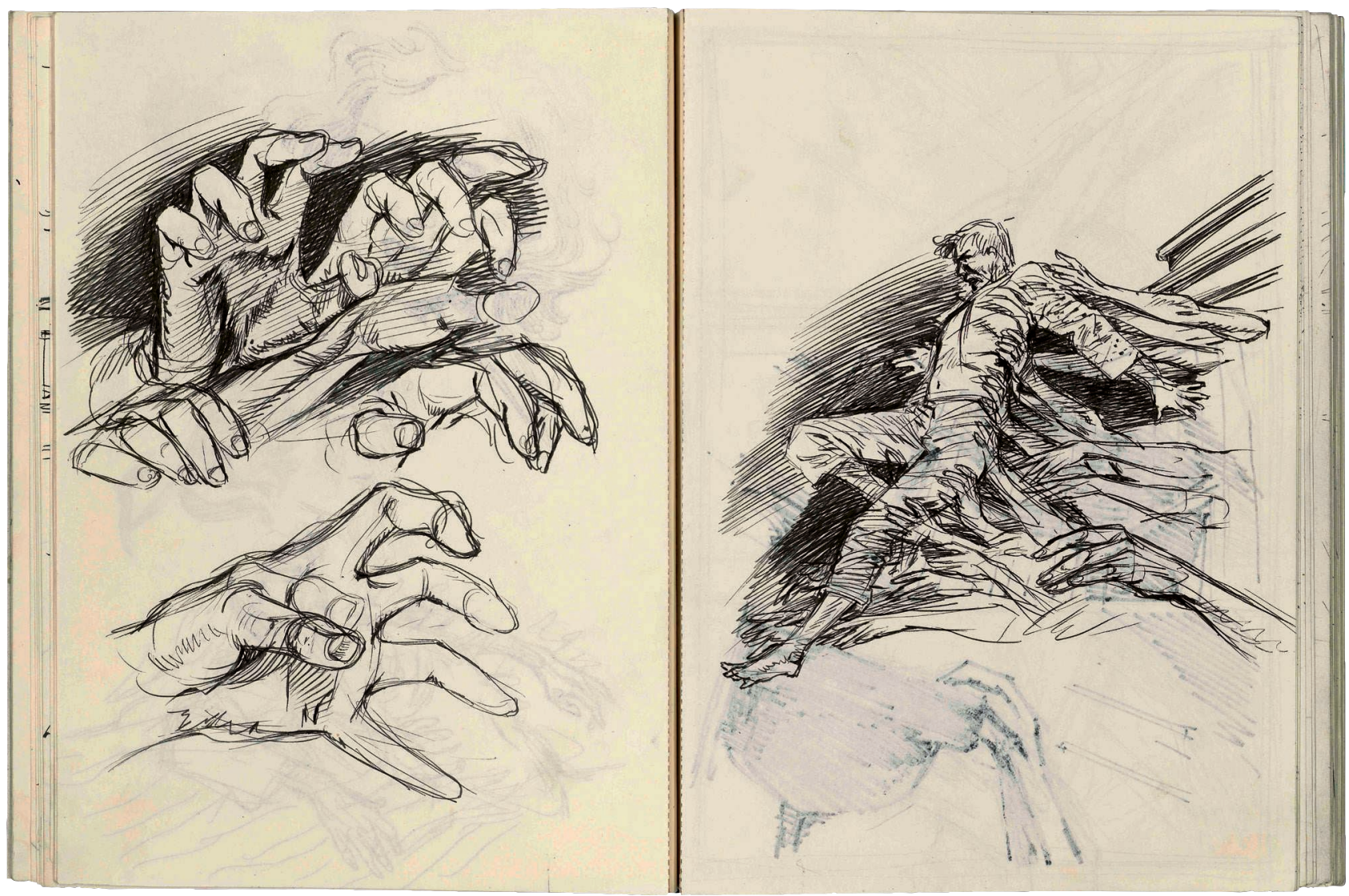

Fig. 5 : François Schuiten, esquisse pour L'Ombre d'un homme (Bibliothèque nationale de France, don des auteurs) 